\title{
Uniaxial Strain Controlling Magnetic Anisotropy in (Ga,Mn)As
}

\author{
J. Zemen ${ }^{a}$, T. JUngWirth ${ }^{a, b}$, J. WUnderlich ${ }^{c}$ \\ AND B.L. Gallagher ${ }^{b}$ \\ ${ }^{a}$ Institute of Physics of the ASCR v. v. i. \\ Cukrovarnicka 10, 16253, Prague 6, Czech Republic \\ ${ }^{b}$ School of Physics and Astronomy, University of Nottingham \\ Nottingham, NG7 2RD, UK \\ ${ }^{c}$ Hitachi Cambridge Laboratory, Cambridge CB3 0HE, UK
}

\begin{abstract}
A theoretical study of magnetocrystalline anisotropy controlled by in-plane lattice symmetry distortions is presented. The uniaxial strain, only presumed by earlier studies in order to model the observed uniaxial magnetic anisotropy, has recently become an experimentally accessible parameter. We show that the lithographically induced strain can compete with intrinsic symmetry breaking, easy axes can take general in-plane directions, and anisotropy fields scale linearly with strain for typical experimental strain magnitudes. Our results are in qualitative agreement with experimental results.

PACS numbers: 75.30.Gw, 78.55.Cr, 75.50.Pp
\end{abstract}

\section{Introduction}

For typical doping levels $1-10 \%$ of Mn magnetic moments, the magnetic dipole interactions are 10-100 times weaker in $(\mathrm{Ga}, \mathrm{Mn}) \mathrm{As}$ than in conventional ferromagnets. Despite the low saturation magnetisation the magnetic anisotropy fields reach $100 \mathrm{mT}$ due to the large spin-orbit coupling induced magnetocrystalline anisotropy. Numerous previous studies have shown that magnetocrystalline anisotropy in (Ga,Mn)As is tunable by changing the level of Mn doping, hole density, temperature, or growth strain [1-4]. Recently, the possibility of breaking the in-plane symmetry of a sample using post-growth patterning has been demonstrated [5-7]. In this work we theoretically investigate the effect of lithographically induced uniaxial strain on magnetic anisotropy of narrow (Ga,Mn)As stripes grown on GaAs substrate for a range of hole densities and typical experimental geometries. 
Our microscopic calculations of the magnetisation angle dependent total energies combine the six-band $k \cdot p$ description of the GaAs host valence band with kinetic-exchange coupling of itinerant holes to the local $\mathrm{Mn}_{\mathrm{Ga}} d^{5}$-moments. For detailed description of how the strain effects are accounted for within this model we refer to Refs. [8-10].

TABLE

Investigated geometries.

\begin{tabular}{c|c|c|c|c|c|c}
\hline \hline & Aligned to & $r$ & $e_{x y}^{\text {intrinsic }}[\%]$ & $e_{x y}^{\text {induced }}[\%]$ & $e_{x x}[\%]$ & $e_{y y}[\%]$ \\
\hline Bar1 & {$[\overline{1} 10]$} & 20 & 0.01 & 0.05 & -0.25 & -0.25 \\
Bar2 & {$[\overline{1} 10]$} & 40 & 0.01 & 0.03 & -0.27 & -0.27 \\
Bar3 & {$[110]$} & 40 & 0.01 & -0.03 & -0.27 & -0.27 \\
Bar4 & {$[110]$} & 20 & 0.01 & -0.05 & -0.25 & -0.25 \\
Bar 5 & {$[100]$} & 40 & 0.01 & 0 & -0.3 & -0.24 \\
Bar6 & {$[100]$} & 20 & 0.01 & 0 & -0.3 & -0.2 \\
Bulk & & & 0.01 & 0 & -0.3 & -0.3
\end{tabular}

We calculate magnetic properties of six thin (Ga,Mn)As stripes on a GaAs substrate. These bars (listed in Table) differ in ratio $r$ of the bar width and thickness and in direction with respect to the crystal axes. For bars 1, 4, and 6 $r=20$, whereas for bars 2, 3, and $5 r=40$, therefore, their relaxation is weaker. All samples share the same "intrinsic" shear strain used commonly to model the experimentally observed uniaxial anisotropy present in bulk samples $[2,3,7]$. The level of $\mathrm{Mn}$ doping is $x=5 \%$ for all bars which corresponds to $f \equiv\left(a_{\mathrm{GaMnAs}}-a_{\mathrm{GaAs}}\right) / a_{\mathrm{GaAs}} \approx 0.3 \%$ (as-grown sample). The relaxation of the growth induced stress in the $(\mathrm{Ga}, \mathrm{Mn})$ As layer allowed by in-plane patterning leads to inhomogeneous strain distribution through the sample. We find the
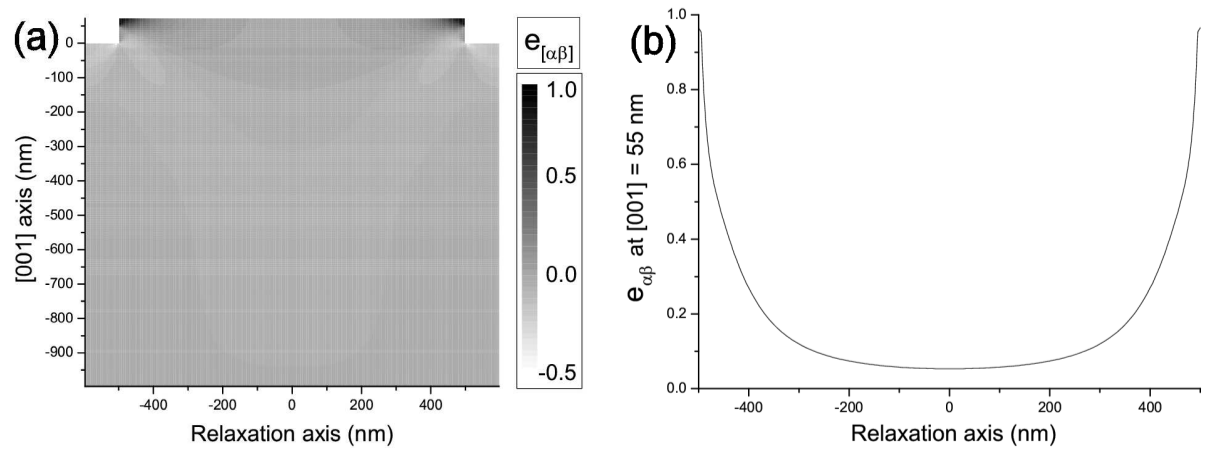

Fig. 1. (a) Strain distribution in $25 \mathrm{~nm}$ thick, $1 \mu \mathrm{m}$ wide (Ga,Mn)As stripe (length of stripe is suppressed, upper corners were removed lithographically). (b) Cut through the relaxation plane at $5 \mathrm{~nm}$ from the bottom of the doped layer. 
average strain using a macroscopic elastic theory simulation implemented by Comsol Mutiphysics (www.comsol.com), which is used as an input parameter for the microscopic band structure calculation. This procedure is justified by the linear dependence of the magnetic anisotropy on local strain (for small - experimentally achievable strain magnitude).

All strain tensor components scale linearly with $f$. Owing to isotropic modelling of $(\mathrm{Ga}, \mathrm{Mn})$ As elastic properties, we can rotate the resulting strain distribution $e_{\alpha \beta}$ presented in Fig. 1 from the "relaxation" coordinates to crystal coordinates (axes [100]-[010]-[001]). The input parameters of the microscopic calculations are then strain components $e_{i j}$, related to the fully relaxed cubic (Ga,Mn)As lattice. The transformation can then be written as: $e_{x y}=f\left(A e_{\alpha \beta} A^{\mathrm{T}}-1\right)$, where matrix $A$ represents a rotation by angle $\theta$ about the [001]-axis.

\section{Results}

We investigate reorientations of in-plane magnetic easy axes as a function of lattice relaxation for a range of hole densities. Figures 2-4 show our results for three selected hole densities, all six considered bars and a bulk sample with no additional relaxation (for reference). Easy direction has the lowest total energy $E_{[\phi]}$.
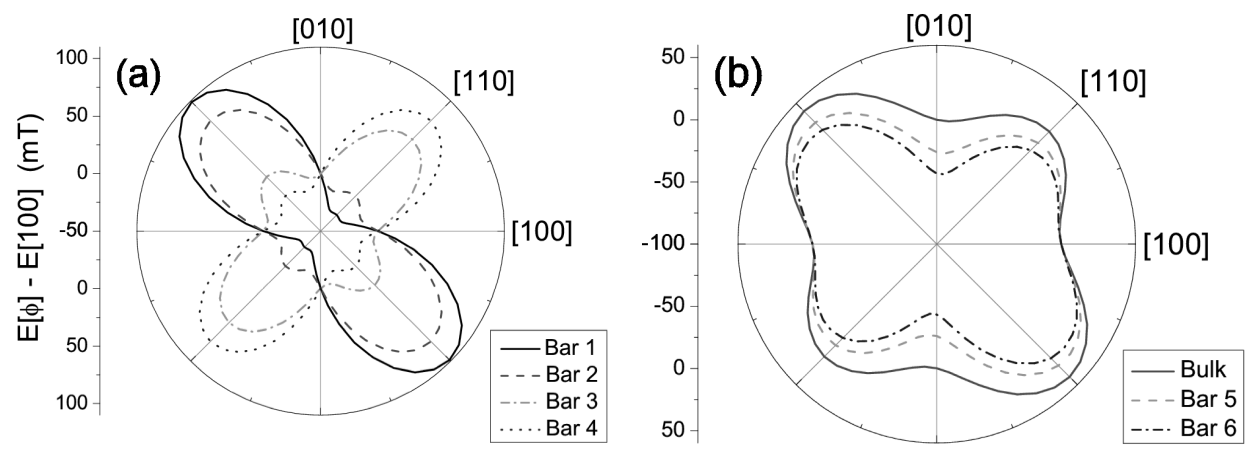

Fig. 2. In-plane anisotropy fields for 6 bars and a hole density of $0.3 \times 10^{20} \mathrm{~cm}^{-3}$ : (a) only diagonal uniaxial strain; (b) intrinsic shear strain and $e_{x x}<e_{y y}$.

Figure 4a shows the presence of two easy axes nearing the main crystal axes for weak relaxation (e.g. bar 3). These axes rotate towards the diagonal that is aligned with their respective bars as the shear strain is increased (e.g. bar 4). This behaviour applies to typical hole densities in $5 \%$ doped as-grown samples and is in agreement with experimental results in Ref. [7]. At low hole density (see Fig. 2a), however, the easy axes rotate towards the diagonal perpendicular to the bar. For all studied hole densities, a strong enough induced strain (roughly $\left|e_{x y}\right|>0.1 \%$ ) results in a single easy axis aligned with certain diagonal. Let us note that there is an intermediate region of hole densities (see Fig. 3a, b) where the uniaxial anisotropy prevails even for low shear strain. 

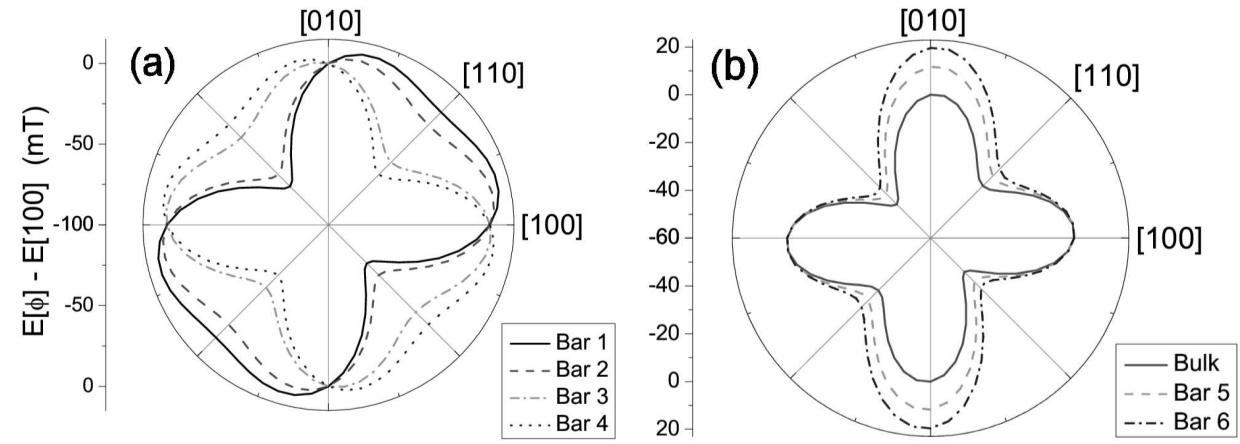

Fig. 3. As in Fig. 2, but for a hole density of $1.3 \times 10^{20} \mathrm{~cm}^{-3}$.
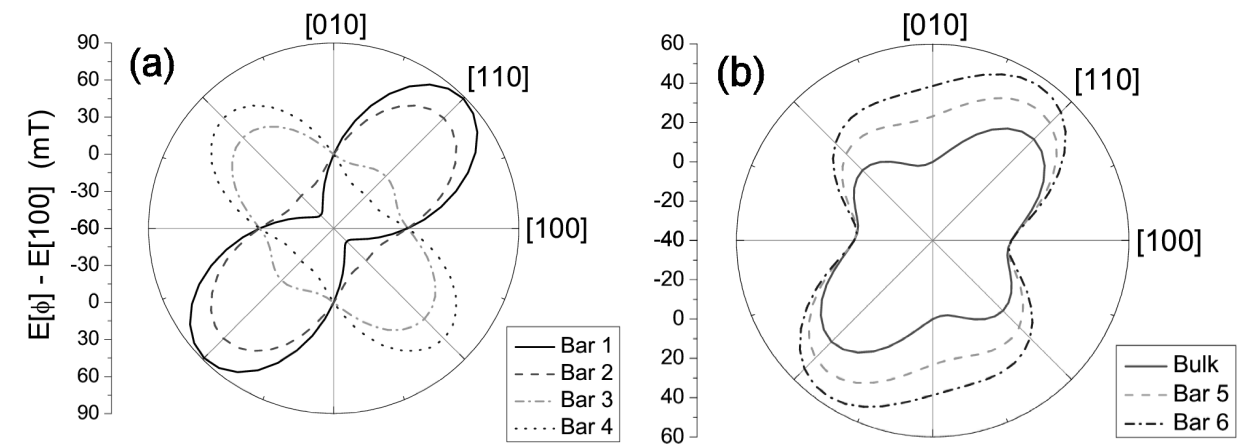

Fig. 4. As in Fig. 2, but for a hole density of $3.3 \times 10^{20} \mathrm{~cm}^{-3}$.

Figure $4 \mathrm{~b}$ reveals the disappearance of the easy axis close to [010] direction, originally present in the bulk sample, upon relaxation along [010] direction. This effect was observed experimentally in Refs. [5, 6]. Again, opposite behaviour occurs at low hole densities (see Fig. 2b), where the [010] easy axis is enhanced with increasing relaxation (decreasing $e_{y y}$ ).

Finally, we point out that our model assumes systems which are far on the metal side of the metal-insulator transition. The lower hole-density regime discussed above, however, would in real (Ga,Mn)As materials correspond to systems with strong hole localisation. Near the metal-insulator transition, where ferromagnetism itself disappears eventually, our model loses its reliability. To conclude, we have shown that lithographically achievable in-plane lattice distortions can break the original bulk symmetry and account for the observed reorientation of magnetic easy axes.

\section{Acknowledgments}

We acknowledge financial support from the EU (NANOSPIN, FP6-2002IST-015728). 


\section{References}

[1] H. Ohno, D. Chiba, F. Matsukura, T. Omiya, E. Abe, T. Dietl, Y. Ohno, K. Ohtani, Nature 408, 944 (2000).

[2] M. Sawicki, K.Y. Wang, K.W. Edmonds, R.P. Campion, C.R. Staddon, N.R.S. Farley, C.T. Foxon, E. Papis, E. Kamińska, A. Piotrowska, T. Dietl, B.L. Gallagher, Phys. Rev. B 71, 121302 (2005).

[3] K.Y. Wang, M. Sawicki, K.W. Edmonds, R.P. Campion, S. Maat, C.T. Foxon, B.L. Gallagher, T. Dietl, Phys. Rev. Lett. 95, 217204 (2005).

[4] L. Thevenard, L. Largeau, D. Mauguin, G. Patriarche, A. Lemaître, N. Vernier, J. Ferré, Phys. Rev. B 73, 195331 (2006).

[5] J. Wenisch, C. Gould, L. Ebel, J. Storz, K. Pappert, M.J. Schmidt, cond-mat/0701479.

[6] S. Hümpfner, K. Pappert, J. Wenisch, K. Brunner, C. Gould, G. Schmidt, L.W. Molenkamp, M. Sawicki, T. Dietl, Appl. Phys. Lett. 90, 102102 (2007).

[7] J. Wunderlich, A.C. Irvine, J. Zemen, V. Holý, A.W. Rushforth, E. De Ranieri, U. Rana, K. Vlýborný, Jairo Sinova, C.T. Foxon, R.P. Campion, D.A. Williams, B.L. Gallagher, T. Jungwirth, to be published in Phys. Rev. B.

[8] T. Dietl, H. Ohno, F. Matsukura, Phys. Rev. B 63, 195205 (2001).

[9] M. Abolfath, T. Jungwirth, J. Brum, A.H. MacDonald, Phys. Rev. B 63, 054418 (2001)

[10] W.W. Chow, S.W. Koch, Semiconductor-Laser Fundamentals, Springer, Berlin 1999. 